\title{
Cardiac intervention rates for older patients with acute myocardial infarction in the United States and Ontario, 2003-2013: a retrospective cohort study
}

\author{
Laura C. Yasaitis PhD, Jun Guan MSc, Dennis T. Ko MD MSc, Amitabh Chandra PhD, \\ Therese A. Stukel PhD
}

\section{Abstract}

Background: Previous work showed lower cardiac intervention rates for patients with acute myocardial infarction (AMI) in Ontario than in the United States. We assessed whether Ontario's efforts to improve access to rapid percutaneous coronary intervention (PCI) for AMI were associated with improved timeliness of care and whether this closed the gap between the 2 jurisdictions.

Methods: In this retrospective cohort study, we followed adults aged 66-99 years in the US and Ontario for 30 days after admission for incident AMI between 2003 and 2013 using health administrative data from both countries. We calculated the proportion of patients who received cardiac catheterization, $\mathrm{PCl}$ and coronary artery bypass grafting on the day of and within 30 days of admission overall and according to AMI type (ST-segment elevation AMI [STEMI] v. non-STEMI) and risk group (low, medium or high predicted risk of 30-d mortality).

Results: We followed 414216 patients in the US and 112484 in Ontario. The large disparities in cardiac intervention rates observed in 2003 mostly disappeared over time. By 2013, the proportion of patients who received same-day PCI was only slightly higher in the US than in Ontario (22.3\% v. 19.2\%), whereas the converse was true for 30-day PCI (44.0\% v. 41.3\%). In 2013, patients with STEMI in the US and Ontario received $\mathrm{PCl}$ at nearly identical rates on the day of admission $(66.3 \% \mathrm{v} .63 .8 \%)$; however, more patients at high risk with STEMI in the US than in Ontario received PCl, both on the day of admission (55.5\% v. 44.7\%) and by 30 days (60.5\% v. 55.0\%).

Interpretation: Despite differences in resources and organization of delivery systems, by 2013 , timely receipt of PCl by Ontario patients with AMI lagged only slightly behind that by US patients. A higher supply of PCI centres in the US may have facilitated earlier intervention among patients at high risk with STEMI.

$\checkmark$ ardiovascular disease is responsible for substantial morbidity and mortality. Invasive cardiac interventions, including percutaneous coronary intervention (PCI), increased rapidly in the United States and Canada between 1993 and 2001 for patients with acute myocardial infarction (AMI) but remained higher in the US than in Ontario. ${ }^{1-9}$ By 2003, evidence from trials showed the superiority of PCI over fibrinolytic therapy for ST-segment elevation AMI (STEMI), with the greatest benefit occurring immediately after symptom onset. ${ }^{10,11}$ Further studies showed the benefit of timely PCI for patients with non-STEMI, particularly patients at high risk. ${ }^{12-17}$ Studies showed the feasibility of transferring patients with STEMI for PCI. ${ }^{18-20}$ Rapid reperfusion became guideline-recommended care in both countries. ${ }^{10,21}$

Although the number of PCI laboratories in Ontario increased from 12 in 2003 to 16 in 2012 (CorHealth Ontario: personal communication, 2016) (1.2 per million residents), the number of PCI-capable sites remained higher in the US (7 per million residents by 2011). ${ }^{22}$

To see whether Ontario's efforts to improve rapid PCI for AMI were associated with improved timeliness of care and whether this closed the gap with results from the US, we examined trends in timing of PCI for older patients with AMI, especially those with STEMI, using health administrative data from the US and Ontario.

\section{Methods}

\section{Setting}

We examined invasive cardiac care provided to patients with incident AMI in the US and Ontario between 2003 and 2013. Health care is universally available to residents of Ontario (population aged $\geq 65$ yr 2 million in $2013^{23}$ ) of all ages, whereas Medicare is a single-payer insurance system that is

Competing interests: None declared.

This article has been peer reviewed.

Correspondence to: Therese Stukel, stukel@ices.on.ca

CMAJ Open 2020. DOI:10.9778/cmajo.20190190 
universally available to all residents of the US aged 65 years or more (44 million in $2013^{24}$ ) and covers invasive cardiac procedures. Medicare patients can choose to receive coverage via traditional Medicare, which reimburses providers on a feefor-service basis, or through a managed care plan or health maintenance organization. During this period, $72 \%-87 \%$ of Medicare enrollees representing most areas of the US had fee-for-service coverage. ${ }^{25}$

\section{Study cohorts and design}

Study patients were US and Ontario residents admitted to an acute care hospital with a first (index) admission for AMI between Jan. 1, 2003, and Dec. 31, 2013. To capture incident admissions, we excluded patients admitted for AMI during the previous year. We therefore restricted enrolment to adults aged 66-99 years, since US Medicare covers patients aged 65 or more. We excluded those with a stay of less than 1 day. We included the first eligible admission and followed patients for 30 days after the index admission date.

For the US, the incident AMI was defined as the first inpatient claim with primary International Classification of Diseases, 9th Revision diagnosis code 410 (except 410.x2, previous AMI) (positive predictive value 0.94). ${ }^{26}$ For Medicare beneficiaries who receive care under a managed care plan or health maintenance organization, the full claims history is not available. To assess comorbidities within the previous year, we excluded patients with less than 12 months of fee-for-service Medicare coverage before their index event. For patients who switched into health maintenance organization plans within 30 days of their index admission $(0.25 \%)$, we noted the date of coverage change.

For Ontario, we defined the incident AMI as the first hospital admission with most responsible International Statistical Classification of Diseases and Related Health Problems, 10th Revision (Canadian version) diagnosis code I21 between Apr. 1, 2003, and Dec. 31, 2013. This approach has been shown to have good performance in identifying AMI and AMI subtype (positive predictive value 0.87$).{ }^{27,28}$ To assess prior comorbidities, we excluded patients with less than 12 months of Ontario Health Insurance Plan eligibility before the index admission. We also excluded admissions where AMI was an in-hospital complication.

For both jurisdictions, we created an index episode of care beginning at initial admission and ending at final discharge, incorporating transfers. The index admission could not be a transfer. For the US, we identified transfers as a hospital admission whose date matched the discharge date of a previous hospital admission, or where the admission source indicated a transfer. For Ontario, we used a 12-hour rule to distinguish transfers from readmissions: an admission that occurred less than 12 hours after a hospital discharge was considered a transfer.

\section{Outcomes}

The primary outcomes were invasive cardiac procedures, specifically, cardiac catheterization, PCI and coronary artery grafting on the day of admission, and 3 and 30 days after the index admission. Procedure codes are provided in Appendix 1,
Supplemental Tables S1 and S2 (available at www.cmajopen. $\mathrm{ca} /$ content/8/2/E437/suppl/DC1).

\section{Covariates}

We identified comorbidities from all hospital admissions during the 12 months before and during the index admission. These included 10 cardiovascular risk factors and 11 comorbidities used in a validated model predicting AMI mortality risk. ${ }^{29}$ For the US, we aggregated diagnosis codes to create indicators for selected condition categories. ${ }^{30}$ For Ontario, we created comorbidity groups that closely matched these condition categories.

We determined rurality of patient residence as follows. For the US, we classified the ZIP code of residence as rural or urban based on the ZIP code Rural-Urban Commuting Areas geographic taxonomy. ${ }^{31}$ For Ontario, we measured rurality using the Rurality Index for Ontario, which accounts for population size and travel time, categorized as urban (score of $0-9$ ) or nonurban (score $\geq 10){ }^{32}$

\section{Data sources}

Medicare claims data have been validated in multiple settings and are often used as the gold standard for assessing other sources of health services data. ${ }^{33-35}$ We identified the US cohort using claims from a $20 \%$ random sample of fee-forservice Medicare beneficiaries with information about inpatient, outpatient and physician services. We used MedPAR and carrier claims to identify whether a patient received cardiac catheterization, PCI or coronary artery grafting, and the date performed. We identified patient comorbidities from the first and second diagnosis codes of hospital admissions.

For Ontario, we linked patient records using unique anonymized, encrypted identifiers across multiple Ontario health administrative databases containing information on all publicly insured, medically necessary hospital and physician services. These include the Discharge Abstract Database (hospital admissions, procedures and transfers; includes the most responsible diagnosis for length of stay, secondary diagnosis codes, comorbidities present on admission and complications during the hospital stay), the National Ambulatory Care Reporting System (same-day surgical procedures), the Ontario Health Insurance Plan (physician billings; includes diagnosis codes and procedures) and the Registered Persons Database (patient demographic information and deaths). We identified comorbidities using secondary Discharge Abstract Database diagnosis fields, excluding complications during the index hospital stay. We determined cardiac procedure dates from the Discharge Abstract Database and the National Ambulatory Care Reporting System, supplementing with Ontario Health Insurance Plan billing dates when necessary. Except for AMI subtype, which was not coded in Ontario health administrative data before 2007, no covariates had missing values.

\section{Statistical analysis}

For both cohorts, we developed a baseline patient severity score using logistic regression to predict 30-day mortality, 
incorporating all baseline patient characteristics and comorbidities ( $c=0.73$ and 0.80 for the US and Ontario, respectively). We used mean predicted mortality as a summary measure of baseline patient severity because it incorporated all measured patient risk factors and has been used in other studies. ${ }^{36}$ The 30 -day predicted risk score was categorized as low $(<10 \%)$, medium $(10 \%-20 \%)$ or high $(>20 \%)$.

We calculated the proportion of patients who received each procedure using Kaplan-Meier estimates, separately by jurisdiction. As we could not track the full claims history of US Medicare patients on managed care plans, we censored for health maintenance organization entry (US cohorts) and death.

We compared proportions of procedures to patients within subtypes of AMI (STEMI v. non-STEMI) and within predicted risk groups. Because Ontario did not code type of AMI until 2007, these analyses focused on the later years. In secondary analyses, we compared proportions across patients at high risk according to AMI type and among patients with STEMI residing in urban versus rural settings to assess the potential role that access to PCI facilities may play. Our sample was so large that absolute differences of less than $0.5 \%$ in the smallest subgroup were statistically significant at the $0.1 \%$ level. We have therefore not reported $p$ values and comment on clinically important differences.

\section{Sensitivity analyses}

In sensitivity analyses, we re-estimated the proportions in several ways. We estimated uncensored proportions to examine sensitivity to censoring. Owing to concerns that different distributions of predicted risk between the 2 jurisdictions might bias our estimates, we reweighted the Ontario cohort to closely resemble the US cohort using a nonparametric approach. We determined the risk scores at each Ontario percentile and used the proportion of US patients falling within each risk score interval to reweight the Ontario percentiles; ${ }^{37}$ proportions were re-estimated with these new weights. Finally, owing to potential bias from competing risk of death, we recomputed all proportions using cumulative incidence competing risk estimates. ${ }^{38}$

\section{Ethics approval}

The Ontario component of the study was approved by the Research Ethics Board of Sunnybrook Health Sciences Centre. The US component was approved by the Institutional Review Board of the National Bureau of Economic Research, Cambridge, Mass.

\section{Results}

In the US, of 455816 potential index admissions, 34914 were excluded because they were transfers, and 6686 because the length of stay was less than 1 day. In Ontario, of 131516 potential index admissions, 15765 and 3267, respectively, were excluded for these reasons. During 2003-2013, there were 414216 patients with incident AMI in the US and 112484 in Ontario who met our inclusion criteria. The cohorts had similar age distributions, mean baseline severity (predicted 30-day mortality risk $13.57 \%$ for the US and $13.75 \%$ for Ontario) and proportions of patients at high risk (Table 1). Nearly one-quarter of patients had STEMI. Comorbidity prevalence values were generally similar between the 2 jurisdictions (Table 2).

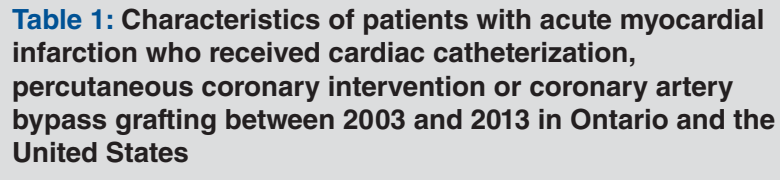

No. (\%) of patients*

$\begin{array}{ccc} & \text { Ontario } & \text { United States } \\ \text { Characteristic } & n=112484 & n=414216\end{array}$

Male sex 60921 (54.2) 207968 (50.2)

Age category, yr

66-70 $20223(18.0) \quad 75202(18.2)$

$71-75 \quad 21584(19.2) \quad 79364(19.2)$

$76-80 \quad 23725(21.1) \quad 83816(20.2)$

81-85 $23306(20.7) \quad 81194(19.6)$

86-90 $15757(14.0) \quad 60094(14.5)$

91-99 $7889(7.0) \quad 34546(8.3)$

Predicted risk of 30-day mortality, \%

$\begin{array}{lll}\text { Mean } \pm \text { SD } & 13.75 \pm 15.04 & 13.57 \pm 10.32\end{array}$

Risk group

\begin{tabular}{|lll|}
\hline Low $(<10 \%)$ & $64374(57.2)$ & $191419(46.2)$ \\
\hline Medium $(10 \%-20 \%)$ & $26930(23.9)$ & $141434(34.1)$ \\
\hline High $(>20 \%)$ & $21180(18.8)$ & $81363(19.6)$
\end{tabular}

Year of index event

\begin{tabular}{|ccc|}
\hline $2003 \dagger$ & $8771(7.8)$ & $48007(11.6)$ \\
\hline 2004 & $11511(10.2)$ & $45845(11.1)$ \\
\hline 2005 & $11111(9.9)$ & $43166(10.4)$ \\
\hline 2006 & $10182(9.1)$ & $39844(9.6)$ \\
\hline 2007 & $10181(9.1)$ & $37622(9.1)$ \\
\hline 2008 & $10689(9.5)$ & $36241(8.8)$ \\
\hline 2009 & $9738(8.7)$ & $33577(8.1)$ \\
\hline 2010 & $10108(9.0)$ & $33651(8.1)$ \\
\hline 2011 & $9877(8.8)$ & $33021(8.0)$ \\
\hline 2012 & $10087(9.0)$ & $32554(7.9)$ \\
\hline 2013 & $10229(9.1)$ & $30688(7.4)$ \\
\hline AMI typeł & & \\
\hline STEMI & $16388(23.9)$ & $100802(24.3)$ \\
\hline Non-STEMI & $50667(74.0)$ & $313414(75.7)$ \\
\hline Missing & $1456(2.1)$ & $0(0.0)$ \\
\hline
\end{tabular}

Note: $\mathrm{AMI}=$ acute myocardial infarction, $\mathrm{SD}$ = standard deviation, STEMI = ST-segment elevation acute myocardial infarction.

*Except where noted otherwise.

†Nine months in Ontario.

¥After 2007 for Ontario $(n=68511)$. 
Table 2: Prevalence of comorbidities

\begin{tabular}{|c|c|c|}
\hline \multirow[b]{2}{*}{ Comorbidity* } & \multicolumn{2}{|c|}{ No. $(\%)$ of patients } \\
\hline & Ontario & United States \\
\hline $\begin{array}{l}\text { History of percutaneous } \\
\text { coronary intervention }\end{array}$ & $944(0.8)$ & $8380(2.0)$ \\
\hline $\begin{array}{l}\text { History of coronary artery } \\
\text { bypass grafting }\end{array}$ & $301(0.3)$ & $1706(0.4)$ \\
\hline $\begin{array}{l}\text { Heart failure (congestive } \\
\text { heart failure) }\end{array}$ & $28993(25.8)$ & $111053(26.8)$ \\
\hline Unstable angina & $4444(4.0)$ & $12112(2.9)$ \\
\hline $\begin{array}{l}\text { Atherosclerosis or other } \\
\text { ischemic heart disease }\end{array}$ & $30064(26.7)$ & $81293(19.6)$ \\
\hline $\begin{array}{l}\text { Shock or cardiorespiratory } \\
\text { failure }\end{array}$ & $8215(7.3)$ & $37850(9.1)$ \\
\hline Valvular heart disease & $2248(2.0)$ & $14038(3.4)$ \\
\hline Hypertension & $8729(7.8)$ & $24822(6.0)$ \\
\hline Cerebrovascular disease & $2980(2.6)$ & $11684(2.8)$ \\
\hline Renal disease & $12216(10.9)$ & 45262 (10.9) \\
\hline $\begin{array}{l}\text { Chronic obstructive } \\
\text { pulmonary disease or } \\
\text { bronchitis }\end{array}$ & $7274(6.5)$ & $30767(7.4)$ \\
\hline Pneumonia & $10501(9.3)$ & $38972(9.4)$ \\
\hline Diabetes & $7415(6.6)$ & $19437(4.7)$ \\
\hline Protein calorie malnutrition & $739(0.7)$ & $2501(0.6)$ \\
\hline Dementia & $2120(1.9)$ & $5354(1.3)$ \\
\hline $\begin{array}{l}\text { Hemiplegia, paraplegia, } \\
\text { paralysis, function disability }\end{array}$ & $1317(1.2)$ & $4397(1.1)$ \\
\hline Peripheral vascular disease & $2772(2.5)$ & $12070(2.9)$ \\
\hline Metastatic cancer & $1237(1.1)$ & $4959(1.2)$ \\
\hline Trauma & $3461(3.1)$ & $12078(2.9)$ \\
\hline Major psychiatric disorder & $1158(1.0)$ & $2299(0.6)$ \\
\hline Acute or chronic liver disease & $520(0.5)$ & $638(0.2)$ \\
\hline \multicolumn{3}{|c|}{$\begin{array}{l}{ }^{*} \text { Includes conditions identified from claims associated with the index admission } \\
\text { and admissions in the previous year except for history of percutaneous coronary } \\
\text { intervention and history of coronary artery bypass grafting, for which only claims } \\
\text { before the index admission were used. }\end{array}$} \\
\hline
\end{tabular}

The large differences in proportions of patients between jurisdictions for all procedures in 2003 diminished over time (Figure 1). By 2013, the proportion of patients who received same-day PCI was only slightly higher in the US than in Ontario $(22.3 \%$ v. $19.2 \%)$, whereas the converse was true for 30 -day PCI $(44.0 \%$ v. $41.3 \%)$. The rates of 3 -day PCI are reported in Appendix 1, Supplemental Table S3. Cardiac catheterization rates mirrored those of PCI. The gap between jurisdictions in 30-day coronary artery grafting rates had decreased by 2013 (9.3\% in the US and 7.8\% in Ontario).

Figure 2 shows PCI rates by AMI type. Although there were substantial differences between the US and Ontario among patients with STEMI in 2007, these had largely disappeared by 2013, when same-day and 30-day proportions were almost identical in the US and Ontario $(66.3 \%$ v. $63.8 \%$, and
$73.2 \%$ v. $76.6 \%$, respectively). In 2013 , the US had higher same-day PCI rates than Ontario among patients with nonSTEMI (11.3\% v. 3.9\%), but the 30 -day rates were almost identical $(33.3 \%$ v. $32.8 \%)$. Three-day rates are reported in Appendix 1, Supplemental Table S4.

We examined relative changes in PCI rates among subgroups of patients by predicted risk (Figure 3). There were large differences between jurisdictions for all risk groups in 2003. By 2013, the differences had narrowed substantially, although US rates remained higher. Among patients at high risk, the same-day PCI rates were $17.6 \%$ in the US and $14.9 \%$ in Ontario, and the corresponding 30 -day rates were $27.7 \%$ and $24.4 \%$. Among patients at low risk, PCI rates were almost identical in the 2 jurisdictions by 2013 .

In secondary analyses, we examined differences among patients at high risk by AMI subtype (Figure 4). Both patients with STEMI and those with non-STEMI received PCI earlier and more frequently in the US than in Ontario, although the rates increased over time in both jurisdictions and the gap had narrowed considerably by 2013. In 2013, the same-day PCI rates among patients with STEMI in the US and Ontario were $55.5 \%$ and $44.7 \%$, respectively, and the corresponding 30 -day rates were $60.5 \%$ and $55.0 \%$. Patients with non-STEMI received PCI earlier and more frequently in the US than in Ontario, with same-day rates in 2013 of $4.9 \%$ and $1.5 \%$, respectively, and 30 -day rates of $17.5 \%$ and $10.4 \%$, respectively.

Finally, we examined PCI rates among patients with STEMI according to urban versus rural residence (Figure 5). Across the study period, PCI rates were higher among urbandwelling patients in Ontario than among those in the US. However, higher proportions of rural-dwelling patients in the US than in Ontario received PCI, especially on the day of admission.

In sensitivity analyses, alternative estimation approaches revealed negligible differences. Unadjusted and adjusted Kaplan-Meier rates were nearly identical. Reweighting the Ontario patient population to have a predicted mortality distribution similar to that of the US made little difference to our findings. Incorporating competing mortality risks had no effect on same-day PCI rates but decreased the rates at 30 days in both the US and Ontario by about 1\% overall and by $1.5 \%$ among patients at high risk.

\section{Interpretation}

Among older patients with AMI, PCI rates were substantially higher in the US than in Ontario in 2003, but the gap had largely closed by 2013, especially for patients with STEMI. Same-day PCI rates increased substantially in both jurisdictions over the study period, likely owing to increased capacity and a greater emphasis on early PCI for patients with STEMI. This suggests that Ontario's policy of increasing PCI capability and staffing, together with its hub-and-spoke model of invasive cardiac care - in which patients with AMI are transported to PCI facilities for same-day procedures and transferred back to their primary hospital as soon as feasible - resulted in timelier access to PCI for patients with STEMI. Among patients with 
A

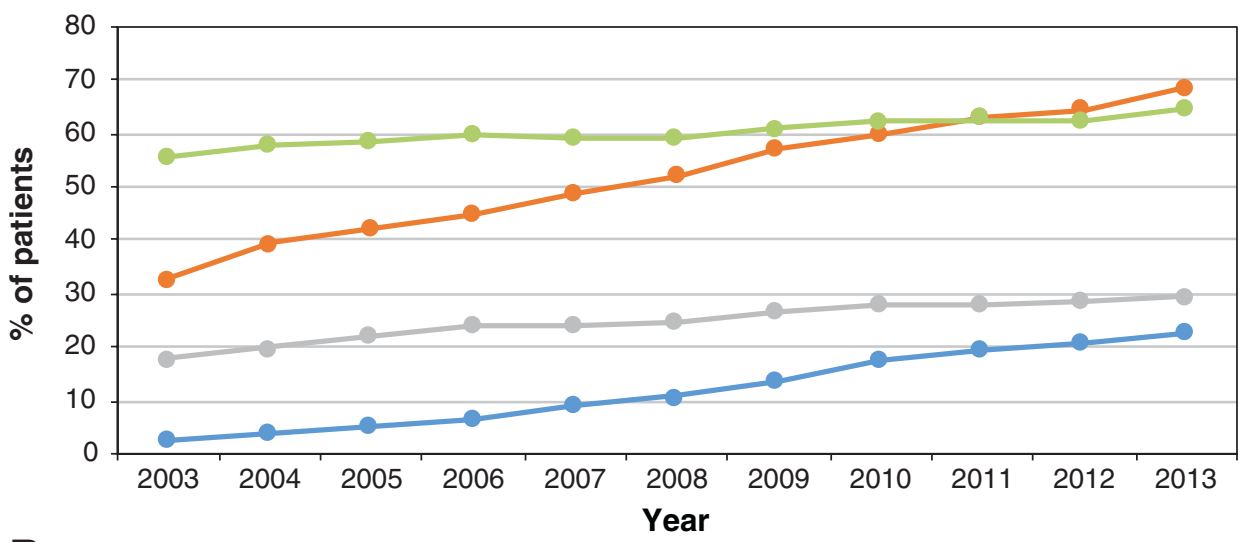

B

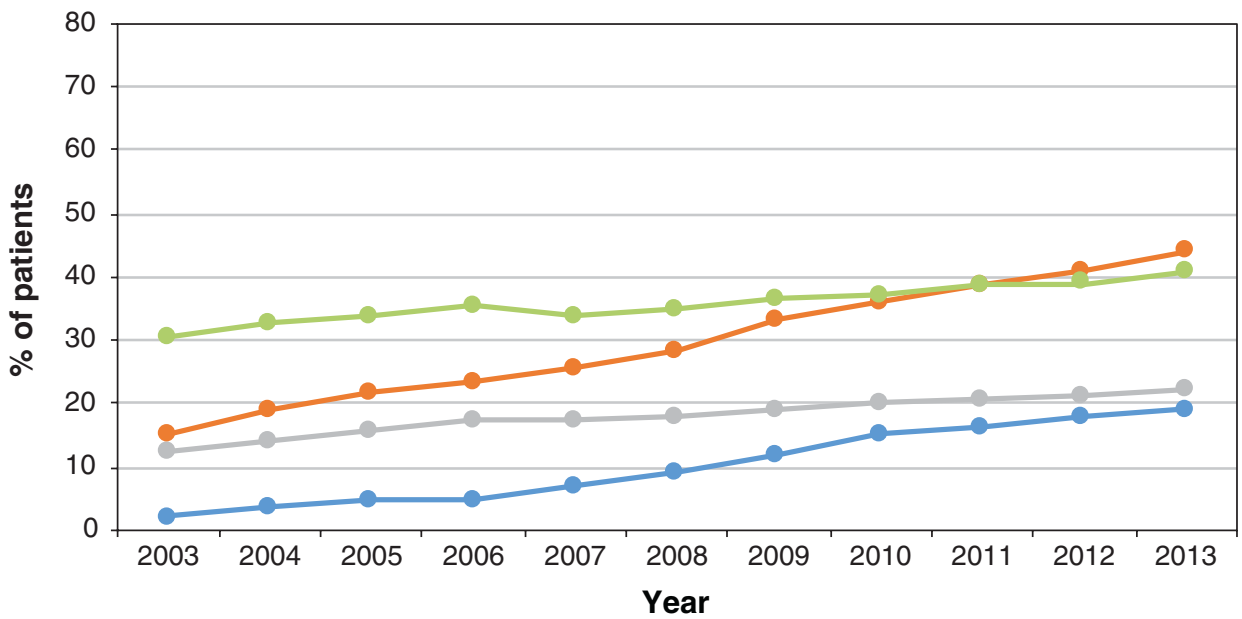

C

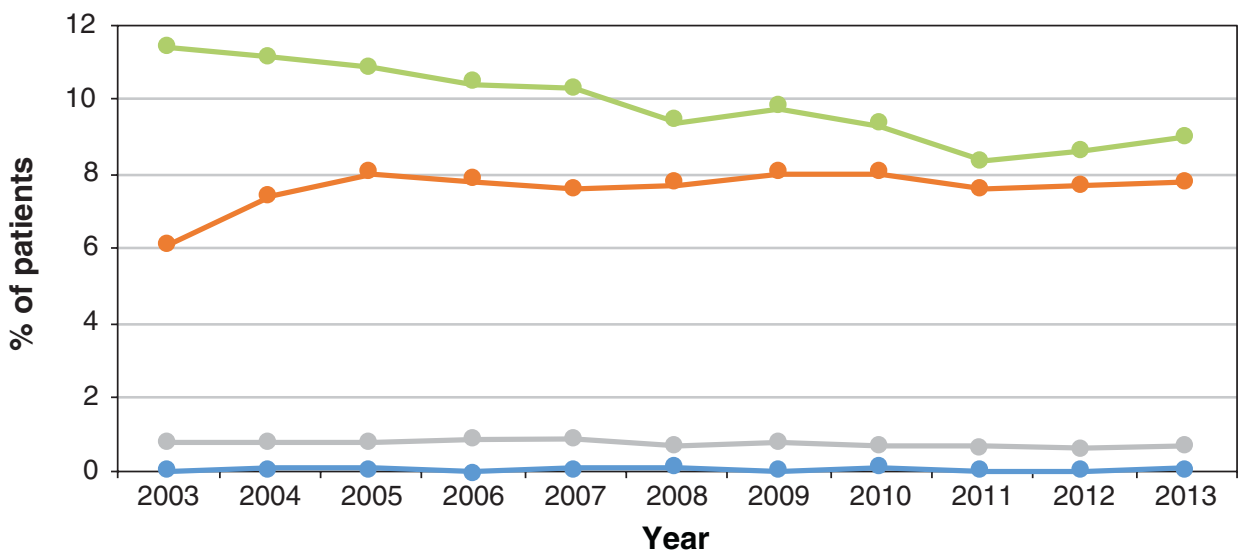

$\longrightarrow$ Ont. same day $\multimap$ US same day $\multimap$ Ont. 30 day $\because$ US day

Figure 1: Rates of cardiac catheterization (A), percutaneous coronary intervention (B) and coronary artery bypass grafting (C) on day of admission for acute myocardial infarction (AMI) and 30 days after AMI. Numbers represent Kaplan-Meier estimates, censoring for death or entry into a managed care Medicare plan (US patients only).

non-STEMI, PCI rates were substantially higher in the US than in Ontario, one of the differences remaining between the 2 jurisdictions in 2013.
Numerous factors may cause PCI rates to be lower than desired. Sicker patients may require care in a cardiac intensive care unit rather than a short-stay unit, and demand for 
A

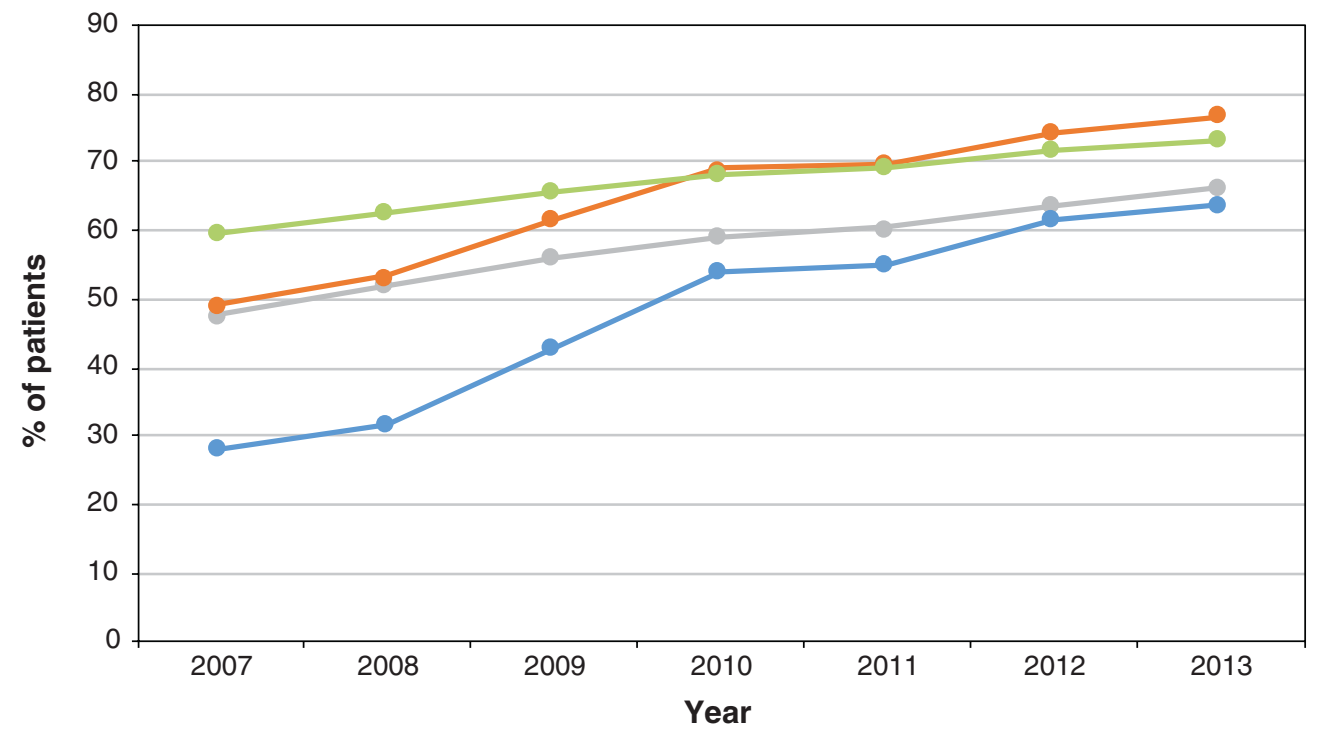

B

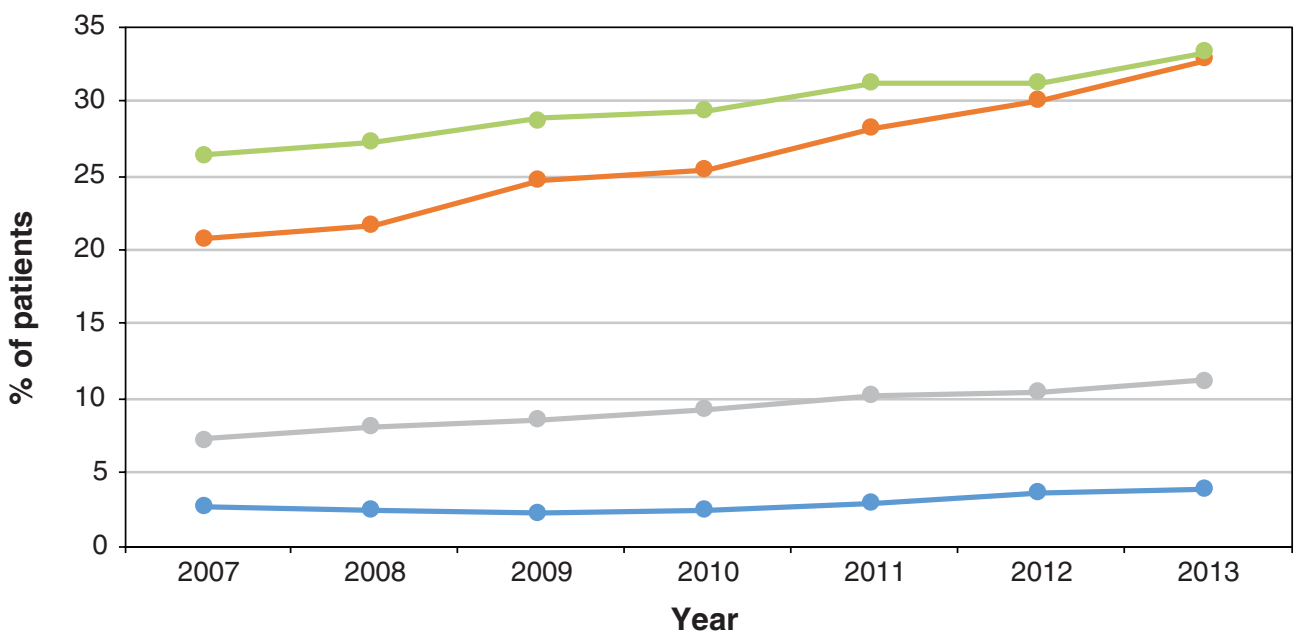

$\multimap$ Ont. same day $\rightleftharpoons$ US same day $\because$ Ont. 30 day $\because$ US 30 day

Figure 2: Percutaneous coronary intervention rates on day of admission for acute myocardial infarction (AMI) and 30 days after AMI for patients with ST-segment elevation AMI (STEMI) (A) and non-STEMI (B). Numbers represent Kaplan-Meier estimates, censoring for death or entry into a managed care Medicare plan (US patients only).

intensive care beds may increase delays in care. In Ontario, because some patients required same-day transportation to a PCI facility, the condition of sicker patients may have been deemed too unstable for transfer. Higher PCI rates among US patients with non-STEMI at high risk likely reflect the greater supply of PCI-capable facilities. Lower PCI rates among rural Ontario patients likely reflect the fact that rural regions are extremely remote, often necessitating helicopter transport of patients to the nearest PCI facility.

Earlier studies showed differences in PCI rates between the US and Canada similar to those found in the present study but did not explore differences by timing of PCI, type of AMI or risk level. Analysis of cardiac procedures over 1992 2001 in the US and Ontario showed that rates for older Ontario patients with AMI lagged behind US rates by 10 years. ${ }^{4-6}$ Ko and colleagues ${ }^{8}$ analyzed cardiac procedures in Ontario and New York State over 1997-2006 and found that the gap in same-day PCI rates among patients with AMI between the 2 jurisdictions narrowed over time but still exhibited a twofold relative difference in 2004-2006.

Cardiac practice has changed substantially since 2013. Centres that perform PCI send most patients with STEMI 
A

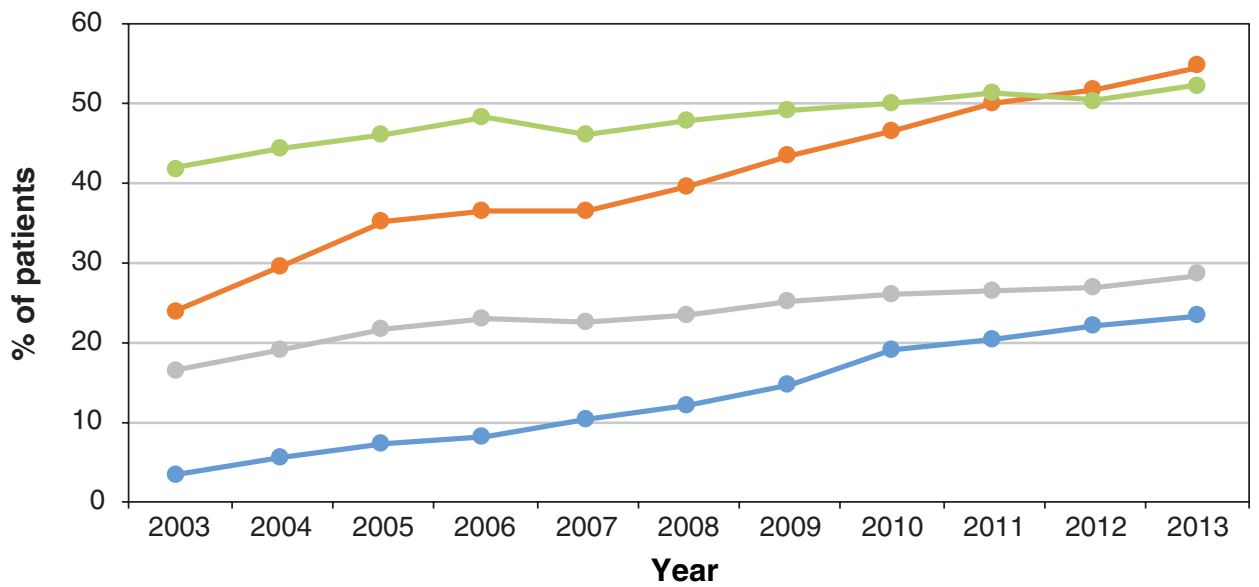

B

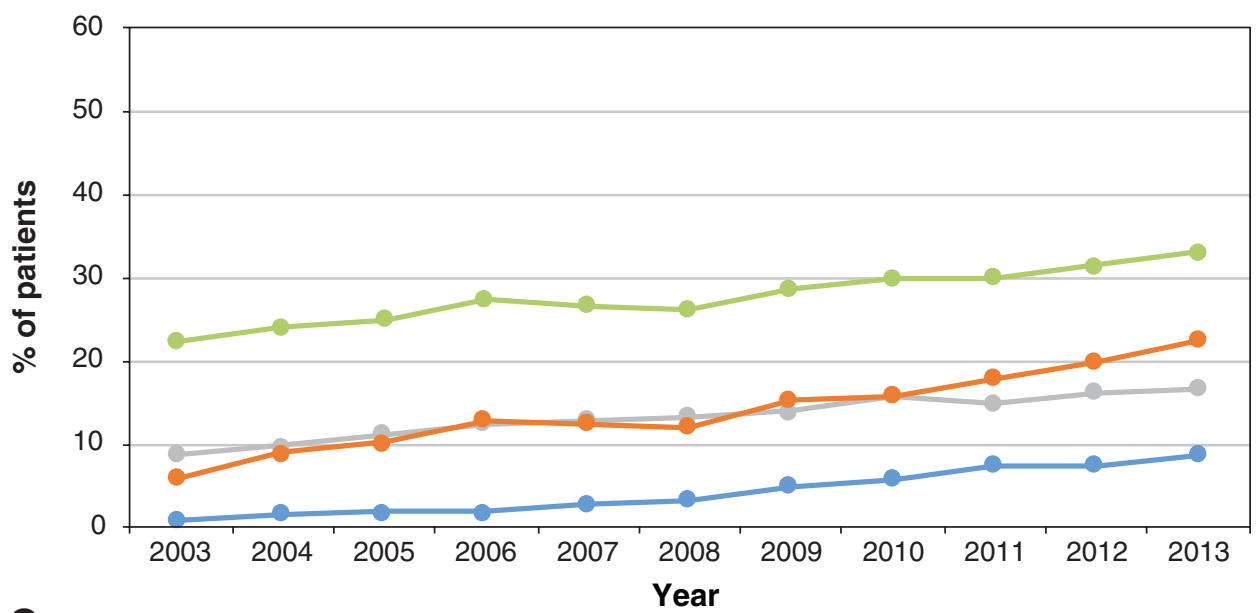

C

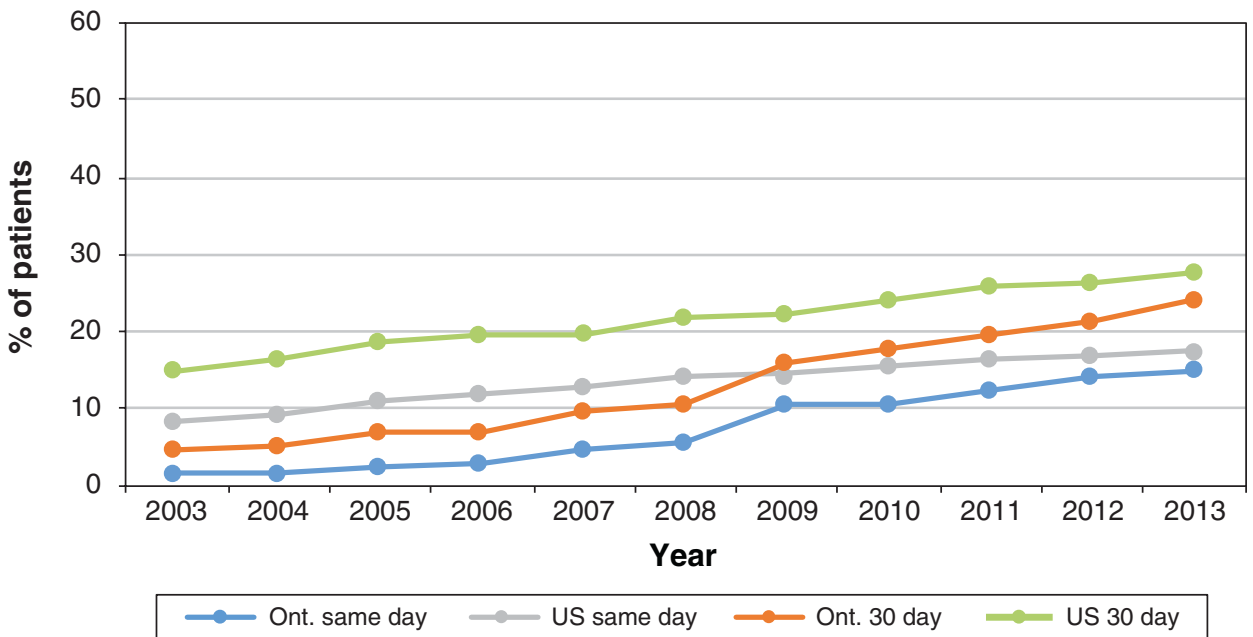

Figure 3: Percutaneous coronary intervention rates on day of admission for acute myocardial infarction (AMI) and 30 days after AMI, by risk category. Risk groups were determined based on predicted 30-day mortality as low $(<10 \%)(A)$, medium $(10 \%-20 \%)(B)$ or high (> 20\%) (C). Numbers represent Kaplan-Meier estimates, censoring for death or entry into a managed care Medicare plan (US patients only). 


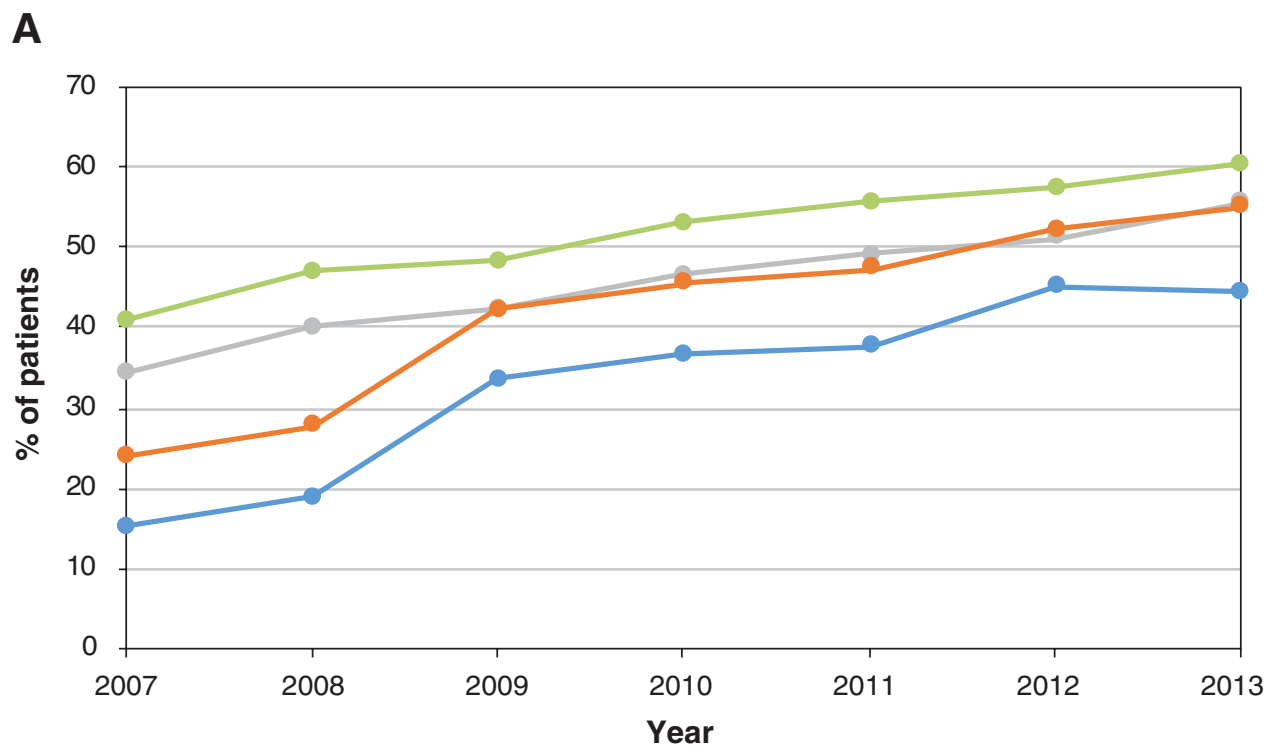

B

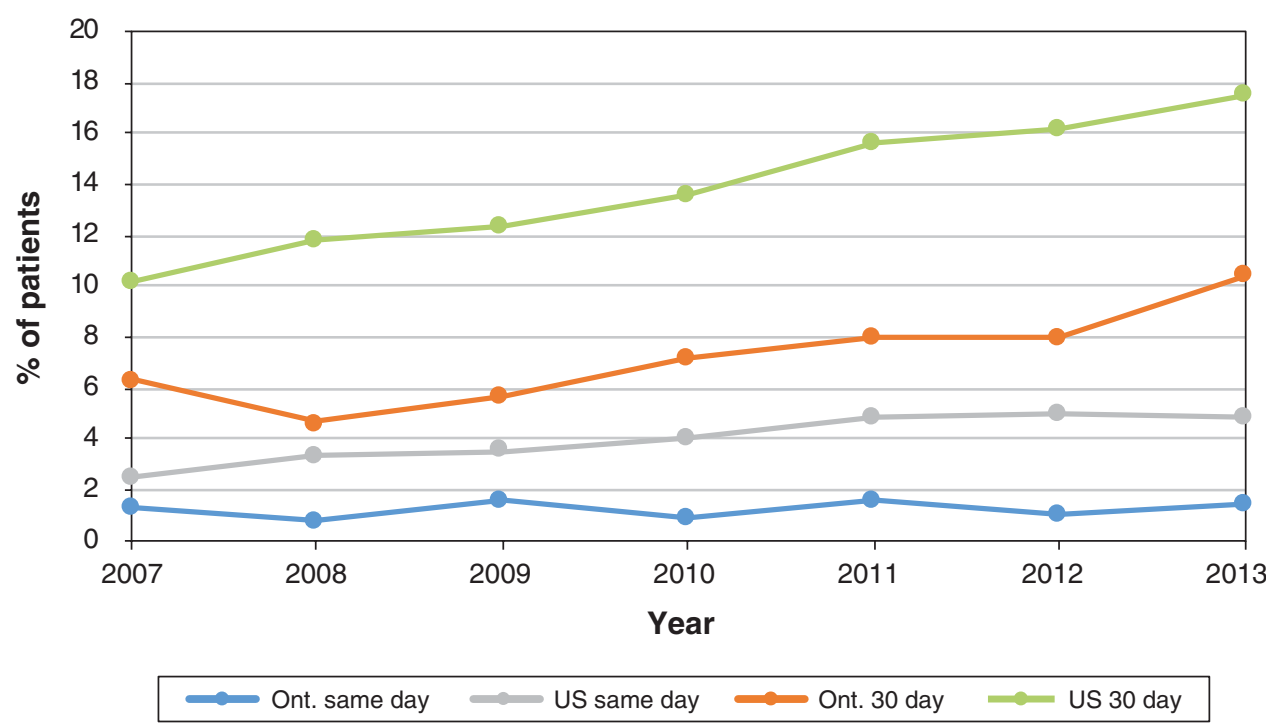

Figure 4: Percutaneous coronary intervention rates on day of admission for acute myocardial infarction (AMI) and 30 days after AMI among patients at high risk with ST-segment elevation AMI (STEMI) (A) or non-STEMI (B). Numbers represent Kaplan-Meier estimates, censoring for death or entry into a managed care Medicare plan (US patients only).

directly to the catheterization laboratory because of the streamlined approach of obtaining an electrocardiographic diagnosis in the ambulance, and coordination among emergency medical services personnel, the emergency department and the cardiac catheterization laboratory. However, onequarter of Canadians do not live within 1 hour of a PCIcapable centre ${ }^{39}$ and often need to be transferred from a nonPCI-capable hospital, which delays care. Transport to hospital by emergency medical services and prehospital electrocardiography are associated with shorter transfer times. ${ }^{39}$

Future research should focus on monitoring primary PCI rates both for patients with STEMI, as the cardiac landscape has changed since 2013, and for those with non-STEMI, given that practice guidelines recommend an invasive strategy within $24-72$ hours. ${ }^{12-17}$ In addition, transfer times from nonPCI-capable to PCI-capable hospitals should be monitored to assess whether patients with AMI are receiving timelier care.

\section{Limitations}

Data use restrictions in both jurisdictions prohibited exporting individual-level data, so we were unable to pool the data into a single analytic data set. As in all studies using administrative claims, data on patients' clinical presentation were limited to recorded diagnoses. Lack of full clinical data and 
A

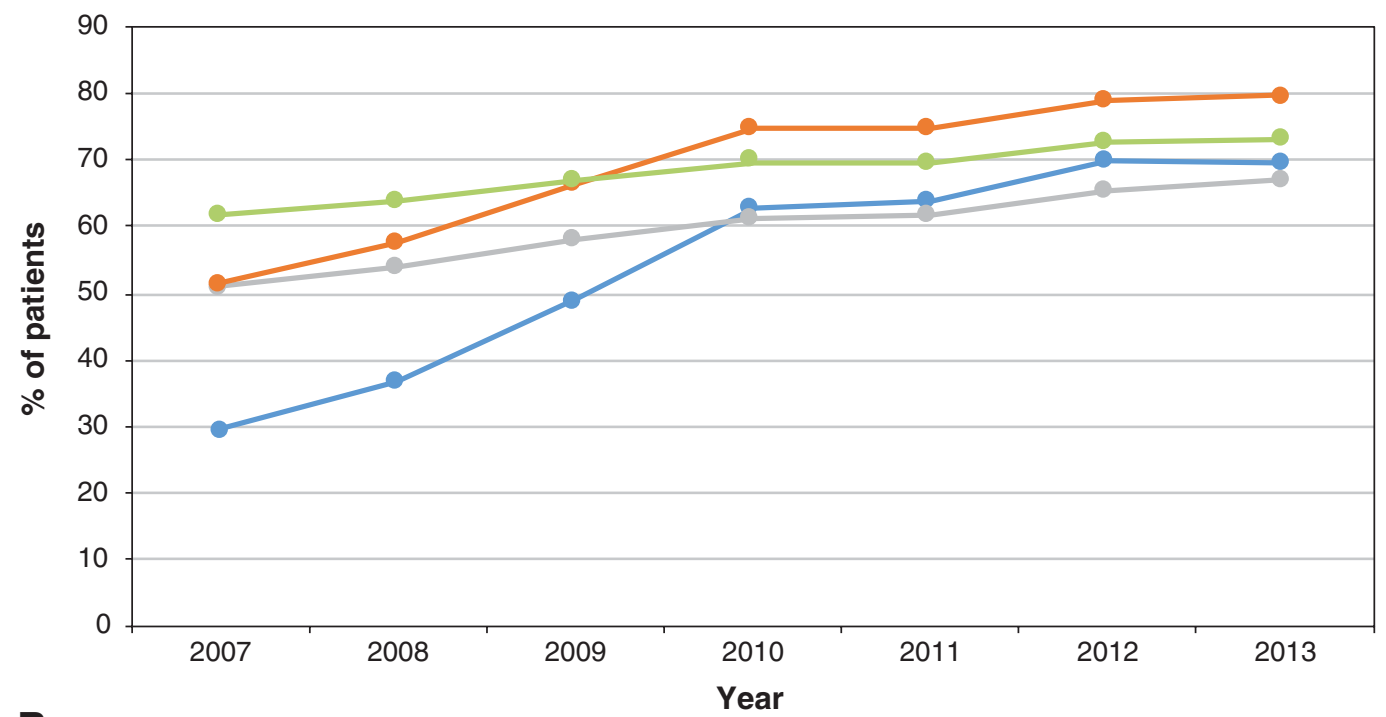

B

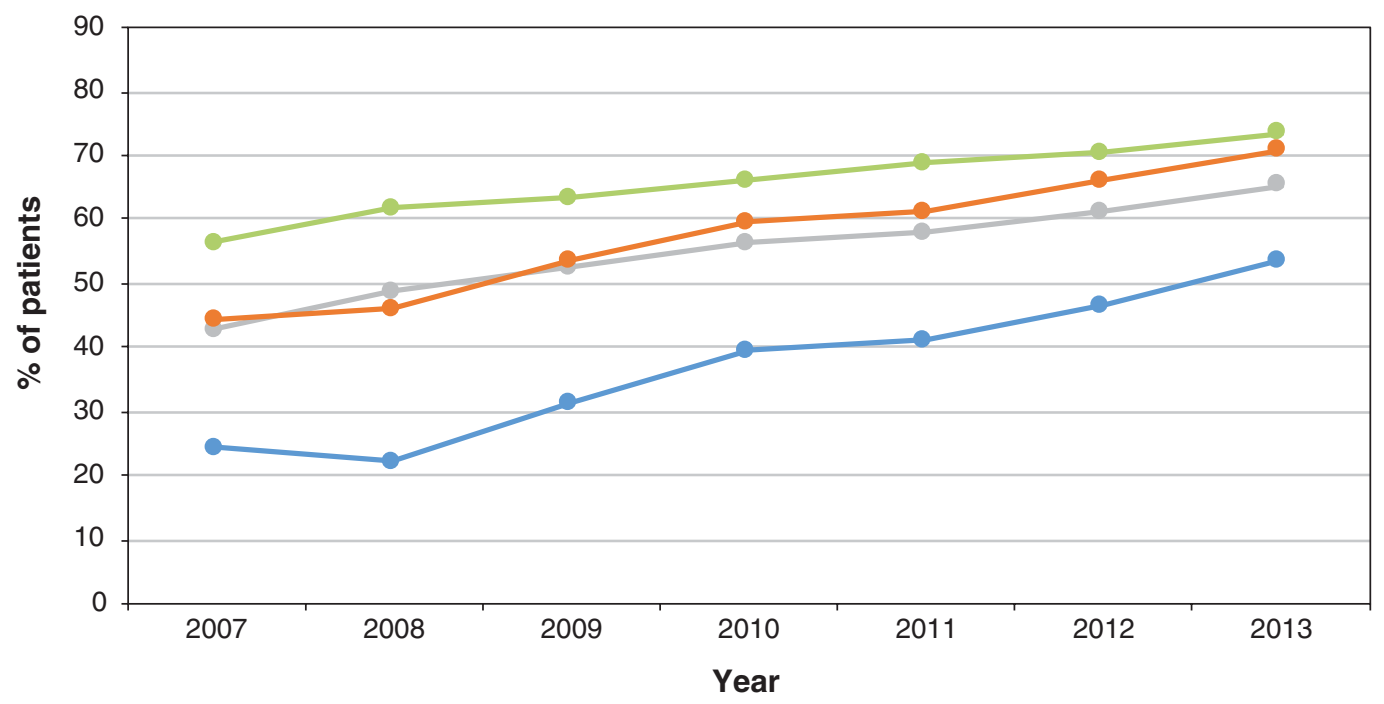

$\because$ Ont. same day $\rightleftharpoons$ US same day 20 day 30 day

Figure 5: Percutaneous coronary intervention rates on day of admission for acute myocardial infarction (AMI) and 30 days after AMI for urban (A) and rural (B) residents with ST-segment elevation AMI. Numbers represent Kaplan-Meier estimates, censoring for death or entry into a managed care Medicare plan (US patients only).

inability to combine data sets precluded us from directly comparing other important outcomes such as mortality. Comparisons of mortality would have required fine adjustment for confounding for unobserved factors such as disease severity, smoking status and clinician judgment regarding who might benefit. Lack of clinical data also prevented us from exploring the appropriateness of care.

In Canada, our study was limited to Ontario, so the findings may not be generalizable to other Canadian provinces; however, given Ontario's size and diversity, and the similar regulation of invasive cardiac resources across the country, the findings likely reflect trends in the rest of the country. Since US Medicare covers only patients aged 65 years or more, the findings apply only to older patients; in addition, they apply only to patients enrolled in fee-for-service Medicare at the index hospital admission. Owing to data use restrictions, we were limited to following US patients through 2013, which precluded more recent follow-up.

Finally, although patients in both Ontario and the US had health insurance and access to treatment, some of the 
differences between the 2 jurisdictions may have been due to sex or socioeconomic disparities, or to unmeasured risk factors across jurisdictions, even among fully insured patients.

\section{Conclusion}

Substantial disparities in 2003 in cardiac intervention rates for AMI, especially STEMI, between the US and Ontario largely disappeared by 2013 despite differences in resources and organization of delivery systems. Higher supply of PCIcapable centres in the US may have facilitated earlier and more interventions, especially among patients whose condition made prompt transfer for treatment difficult.

\section{References}

1. Tu JV, Pashos CL, Naylor CD, et al. Use of cardiac procedures and outcomes in elderly patients with myocardial infarction in the United States and Canada. N Engl f Med 1997;336:1500-5.

2. Fu Y, Chang WC, Mark D, et al. Canadian-American differences in the management of acute coronary syndromes in the GUSTO IIb trial: one-year follow-up of patients without ST-segment elevation. Global Use of Strategies to Open Occluded Coronary Arteries (GUSTO) II Investigators. Circulation 2000;102:1375-81.

3. Kaul P, Armstrong PW, Chang WC, et al. Long-term mortality of patients with acute myocardial infarction in the United States and Canada: comparison of patients enrolled in Global Utilization of Streptokinase and t-PA for Occluded Coronary Arteries (GUSTO)-I. Circulation 2004;110:1754-60.

4. Alter DA, Stukel TA, Newman A. Proliferation of cardiac technology in Canada: a challenge to the sustainability of Medicare. Circulation 2006;113:380-7.

5. Lucas FL, DeLorenzo MA, Siewers AE, et al. Temporal trends in the utilization of diagnostic testing and treatments for cardiovascular disease in the United States, 1993-2001. Circulation 2006;113:374-9.

6. Ayanian JZ. Rising rates of cardiac procedures in the United States and Canada: Too much of a good thing? Circulation 2006;113:333-5.

7. Ko DT, Krumholz HM, Wang Y, et al. Regional differences in process of care and outcomes for older acute myocardial infarction patients in the United States and Ontario, Canada. Circulation 2007;115:196-203.

8. Ko DT, Tu JV, Samadashvili Z, et al. Temporal trends in the use of percutaneous coronary intervention and coronary artery bypass surgery in New York State and Ontario. Circulation 2010;121:2635-44.

9. Ko DT, Tu JV, Austin PC, et al. Prevalence and extent of obstructive coronary artery disease among patients undergoing elective coronary catheterization in New York State and Ontario. FAMA 2013;310:163-9.

10. Antman EM, Anbe DT, Armstrong PW, et al. ACC/AHA guidelines for the management of patients with ST-elevation myocardial infarction: a report of the American College of Cardiology/American Heart Association Task Force on Practice Guidelines (Committee to Revise the 1999 Guidelines for the Management of Patients with Acute Myocardial Infarction). 7 Am Coll Cardiol 2004;44:E1-211.

11. Keeley EC, Boura JA, Grines CL. Primary angioplasty versus intravenous thrombolytic therapy for acute myocardial infarction: a quantitative review of 23 randomised trials. Lancet 2003;361:13-20.

12. Cannon CP, Weintraub WS, Demopoulos LA, et al.; TACTICS (Treat Angina with Aggrastat and Determine Cost of Therapy with an Invasive or Conservative Strategy) - Thrombolysis in Myocardial Infarction 18 Investigators. Comparison of early invasive and conservative strategies in patients with unstable coronary syndromes treated with the glycoprotein $\mathrm{Ilb} / \mathrm{III}$ inhibitor tirofiban. N Engl 7 Med 2001;344:1879-87.

13. Fox KA, Poole-Wilson PA, Henderson RA, et al.; Randomized Intervention Trial of Unstable Angina Investigators. Interventional versus conservative treatment for patients with unstable angina or non-ST-elevation myocardial infarction: the British Heart Foundation RITA 3 randomised trial. Randomized Intervention Trial of unstable Angina. Lancet 2002:360:743-51.

14. Solomon DH, Stone PH, Glynn RJ, et al. Use of risk stratification to identify patients with unstable angina likeliest to benefit from an invasive versus conservative management strategy. 7 Am Coll Cardiol 2001;38:969-76.

15. Braunwald $\mathrm{E}$. Application of current guidelines to the management of unstable angina and non-ST-elevation myocardial infarction. Circulation 2003;108 (Suppl 1):III28-37.

16. Bach RG, Cannon CP, Weintraub WS, et al. The effect of routine, early invasive management on outcome for elderly patients with non-ST-segment elevation acute coronary syndromes. Ann Intern Med 2004;141:186-95.

17. Mehta SR, Granger CB, Boden WE, et al.; TIMACS Investigators. Early versus delayed invasive intervention in acute coronary syndromes. $N$ Engl $7 \mathrm{Med}$ 2009;360:2165-75.
18. Cantor WJ, Fitchett D, Borgundvaag B, et al.; TRANSFER-AMI Trial Investigators. Routine early angioplasty after fibrinolysis for acute myocardial infarction. N Engl f Med 2009;360:2705-18.

19. Le May MR, So DY, Dionne R, et al. A citywide protocol for primary PCI in ST-segment elevation myocardial infarction. N Engl 7 Med 2008;358:231-40.

20. Andersen HR, Nielsen TT, Rasmussen K, et al.; DANAMI-2 Investigators. A comparison of coronary angioplasty with fibrinolytic therapy in acute myocardial infarction. N Engl f Med 2003;349:733-42.

21. Ontario Health Technology Advisory Committee. OHTAC recommendation: primary angioplasty. Toronto: Health Quality Ontario; 2004.

22. Langabeer JR, Henry TD, Kereiakes DJ, et al. Growth in percutaneous coronary intervention capacity relative to population and disease prevalence. $7 \mathrm{Am}$ Heart Assoc 2013;2:e000370.

23. Ontario population projections, 2018-2046. Table 2: Ontario population and selected characteristics under three scenarios, 2011-2046. Queen's Printer for Ontario; 2010 [last modified 2019 Oct. 1]. Available: https://www.fin.gov. on.ca/en/economy/demographics/projections/table2.html (accessed 2020 Apr. 28).

24. 2013 CMS statistics. U.S. Department of Health \& Human Services; 2013. CMS publ. no. 03504. Available: https://www.cms.gov/Research-Statistics-Data -and-Systems/Statistics-Trends-and-Reports/CMS-Statistics-Reference-Booklet/ Downloads/CMS_Stats_2013_final.pdf (accessed 2020 Apr. 28).

25. Neuman T, Casillas G, Jacobson G. Medicare advantage and traditional medicare: Is the balance tipping? Menlo Park (CA): The Henry J. Kaiser Family Foundation; 2015. Available: http://files.kff.org/attachment/issue-brief -medicare-advantage-and-traditional-medicare-is-the-balance-tipping (accessed 2020 Apr. 28).

26. Kiyota Y, Schneeweiss S, Glynn RJ, et al. Accuracy of Medicare claims-based diagnosis of acute myocardial infarction: estimating positive predictive value on the basis of review of hospital records. Am Heart 72004;148:99-104.

27. Juurlink D, Preyra C, Croxford R, et al. Canadian Institute for Health Information Discharge Abstract Database: a validation study. Toronto: Institute for Clinical Evaluative Sciences; 2006.

28. Patel AB, Quan H, Welsh RC, et al. Validity and utility of ICD-10 administrative health data for identifying ST -and non-ST-elevation myocardial infarction based on physician chart review. CMA7 Open 2015;3:E413-8.

29. Krumholz HM, Wang Y, Mattera JA, et al. An administrative claims model suitable for profiling hospital performance based on 30-day mortality rates among patients with an acute myocardial infarction. Circulation 2006;113: 1683-92.

30. Pope GC, Kautter J, Ellis RP, et al. Risk adjustment of Medicare capitation payments using the CMS-HCC model. Health Care Financ Rev 2004;25: $119-41$.

31. Hart G, Cromartie J. Temporary ZIP RUCA 3.10 file access page. Grand Forks (ND): Center for Rural Health, School of Medicine \& Health Sciences, University of North Dakota; 2014. Available: https://ruralhealth.und.edu/ruca (accessed 2018 Aug. 28)

32. Kralj B. Measuring rurality - RIO2008_BASIC: methodology and results. Toronto: Ontario Medical Association; 2009.

33. Leonard CE, Brensinger CM, Nam YH, et al. The quality of Medicaid and Medicare data obtained from CMS and its contractors: implications for pharmacoepidemiology. BMC Health Serv Res 2017;17:304.

34. Lavery JA, Lipitz-Snyderman A, Li DG, et al. Identifying cancer-directed surgeries in Medicare claims: a validation study using SEER-Medicare data. $7 \mathrm{CO}$ Clin Cancer Inform 2019;3:1-24.

35. Noone AM, Lund JL, Mariotto A, et al. Comparison of SEER treatment data with Medicare claims. Med Care 2016;54:e55-64.

36. Stukel TA, Fisher ES, Alter DA, et al. Association of hospital spending intensity with mortality and readmission rates in Ontario hospitals. FAMA 2012;307: $1037-45$.

37. Barsky R, Bound J, Charles KK, et al. Accounting for the black-white wealth gap: a nonparametric approach. 7 Am Stat Assoc 2002;97:663-73.

38. Austin PC, Lee DS, Fine JP. Introduction to the analysis of survival data in the presence of competing risks. Circulation 2016;133:601-9.

39. Shi O, Khan AM, Rezai MR, et al. Factors associated with door-in to doorout delays among ST-segment elevation myocardial infarction (STEMI) patients transferred for primary percutaneous coronary intervention: a population-based cohort study in Ontario, Canada. BMC Cardiovasc Disord 2018;18:204.

Affiliations: Leonard Davis Institute of Health Economics (Yasaitis), University of Pennsylvania, Philadelphia, Pa.; ICES Central (Guan, Ko, Stukel); Department of Medicine (Ko), Schulich Heart Centre, Sunnybrook Health Sciences Centre, and Institute of Health Policy, Management and Evaluation (Ko, Stukel), University of Toronto, Toronto, Ont.; John F. Kennedy School of Government (Chandra), Harvard University; Harvard Business School (Chandra); National Bureau of Economics Research (Chandra), Cambridge, Mass.; The Dartmouth Institute for Health Policy \& Clinical Practice (Stukel), Geisel School of Medicine, Dartmouth College, Hanover, NH 
Contributors: Laura Yasaitis, Dennis Ko, Amitabh Chandra and Therese Stukel conceived the study. Laura Yasaitis, Jun Guan, Amitabh Chandra and Therese Stukel contributed to the study design. Laura Yasaitis and Jun Guan analyzed the data. Therese Stukel, Laura Yasaitis, Dennis Ko and Amitabh Chandra interpreted the data. Laura Yasaitis drafted the manuscript. All of the authors revised the manuscript critically for important intellectual content, approved the final version to be published and agreed to be accountable for all aspects of the work.

Funding: This study was supported by ICES, which is funded by an annual grant from the Ontario Ministry of Health and Long-Term Care. It was also supported through funding provided by Foundation Grant FDN 143303 from the Canadian Institutes of Health Research and by grant PO1 AG019783 from the US National Institute on Aging.

Data sharing: For the Ontario data, the data set from this study is held securely in coded form at ICES. Although legal data-sharing agreements between ICES and data providers (health organizations and government) prohibit ICES from making the data set publicly available, access may be granted to those who meet prespecified criteria for confidential access, available at www.ices.on.ca/DAS. The data set creation plan and analytic code are available from the corresponding author on request, with the understanding that the computer programs may rely on coding templates or macros that are unique to ICES and are therefore inaccessible or may require modification. For the US Medicare data, legal data-sharing agreements between the National Bureau of Economic Research (NBER) and the data provider, the US Centers for Medicare and Medicaid, prohibit NBER from making the data set publicly available. Access may be granted to those who meet prespecified criteria for confidential access, available from the Centers for Medicare and Medicaid via ResDAC at https:// www.resdac.org. The data set creation plan and analytic code are available from the corresponding author on request, with the understanding that the computer programs may rely on coding templates or macros that are unique to NBER and are therefore inaccessible or may require modification.

Disclaimer: This study was supported by ICES, which is funded by an annual grant from the Ontario Ministry of Health and Long-Term Care (MOHLTC). The opinions, results and conclusions reported in this article are those of the authors and are independent from the funding sources. No endorsement by ICES or the Ontario MOHLTC is intended or should be inferred. The funding agencies had no role in the study design; the collection, analysis or interpretation of the data; the writing of the article; or the decision to submit the article for publication.

Supplemental information: For reviewer comments and the original submission of this manuscript, please see www.cmajopen.ca/content/8/2/ E437/suppl/DC1. 